\title{
Particle Simulation of Plume Flows from an Anode-Layer Hall Thruster
}

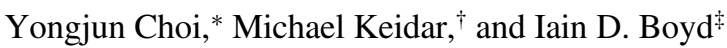 \\ University of Michigan, Ann Arbor, Michigan 48109
}

DOI: $10.2514 / 1.28384$

\begin{abstract}
Two-dimensional axisymmetric simulations of xenon-plasma-plume flowfields from a D55 anode-layer Hall thruster are performed with a hybrid particle-fluid method. In this simulation, the Boltzmann model and a detailed fluid model are used to compute the electron properties, the direct simulation Monte Carlo method models the collisions of heavy particles, and the particle-in-cell-method models the transport of ions in electric fields. The accuracy of the simulation is assessed through comparison with various measured data. The detailed electron fluid model provides improved predictions in comparison with the Boltzmann model in the near and far fields of the plume. Improved correspondence is obtained with the detailed model to all measured data including plasma potential, ion current density, and ion velocity.
\end{abstract}

\section{Nomenclature}

$C_{i}=$ ionization coefficient

$c_{e}=$ mean electron thermal velocity

$\mathbf{E}=$ electric field vector

$e \quad=$ unit charge

$g=$ relative velocity

$\mathbf{j}=$ current density vector

$k=$ Boltzmann constant

$m_{e}=$ electron mass

$m_{i}=$ ion mass

$n_{a}=$ atom number density

$n_{e}=$ electron number density

$n_{\text {ref }}=$ reference plasma number density

$p_{e}=$ electron pressure

$\mathbf{R}=$ friction terms

$T_{e}=$ electron temperature

$T_{H}=$ heavy particle temperature

$\mathbf{V}_{\mathbf{e}}=$ electron velocity vector

$\varepsilon_{i}=$ ionization energy

$\kappa_{e}=$ electron thermal conductivity

$v_{e}=$ electron collision frequency

$v_{\mathrm{ei}}=$ ion-electron collision frequency

$\nu_{\mathrm{en}}=$ neutral-electron collision frequency

$\sigma=$ plasma conductivity

$\sigma_{i}=$ reference cross section for xenon

$\phi \quad=$ plasma potential

$\phi_{\text {ref }}=$ reference plasma potential

$\Psi=$ electron stream function

\section{Introduction}

$\mathbf{H}$ ALL-EFFECT thrusters represent an efficient form of electric propulsion devices for applications requiring low thrust levels

Presented as Paper 5026 at the 42nd AIAA/ASME/SAE/ASEE Joint Propulsion Conference and Exhibit, Sacramento, CA, 9-12 July 2006; received 16 October 2006; accepted for publication 11 December 2007. Copyright $\odot 2008$ by the American Institute of Aeronautics and Astronautics, Inc. All rights reserved. Copies of this paper may be made for personal or internal use, on condition that the copier pay the $\$ 10.00$ per-copy fee to the Copyright Clearance Center, Inc., 222 Rosewood Drive, Danvers, MA 01923; include the code 0748-4658/08 $\$ 10.00$ in correspondence with the CCC.

${ }^{*}$ Graduate Student, Department of Aerospace Engineering; currently Assistant Professor, Mechanical and Aerospace Engineering, George Washington University, Washington, D.C., 20052. Senior Member AIAA.

${ }^{\dagger}$ Assistant Research Scientist, Department of Aerospace Engineering. Senior Member AIAA.

Frofessor, Department of Aerospace Engineering. Associate Fellow AIAA. (e.g., station-keeping, orbit raising, and orbit transfers). In a Hall thruster, ions are accelerated by electric fields and used to generate propulsive thrust. The energy required to accelerate ions is obtained from onboard batteries or solar cells. Hall thrusters are able to perform better than chemical propulsion systems because Hall thrusters can obtain electricity input directly in space through solar cells, do not need to carry any oxidizer, and so allow a larger payload. Furthermore, Hall thrusters can realize much higher propellant exhaust velocities than chemical propulsion systems, thereby achieving higher impulse from a given propellant mass and making the use of Hall thrusters for interplanetary missions feasible.

In the past years, two types of Hall thrusters were developed: a thruster with closed electron drift and extended acceleration zone, or stationary plasma thruster (SPT), and a thruster with a very short acceleration channel, or thruster with anode layer (TAL). The SPT employs a relatively long acceleration channel and ceramic-wallinsulator materials, such as boron nitride or silicon carbide. The TAL employs a shorter acceleration channel and conducting-metallic-wall materials, which are typically stainless steel or molybdenum.

Among Hall thruster technologies, TAL, which was developed in the 1960s at TsNIIMASH, seems to be advantageous for two reasons. First, TAL has a very short acceleration zone (a few millimeters) and so there is less contact of ions with thruster surfaces; hence, it is favorable for long-term missions because of a reduction in erosion of thruster components. Second, higher-power Hall thrusters will be needed in future space missions and a TAL with very high power has been specially developed to meet this requirement [1].

Modeling of the plume fields yields important information in two different ways. First, it provides understanding of the plume impingement that involves fluxes of high-energy ions and chargeexchanged particles onto sensitive spacecraft devices such as solar arrays. Second, plume modeling also helps to clarify the complex plasma processes inside the thruster with the aim of improving propulsion performance. The near-field plume of a Hall thruster is a very important region because its high plasma density makes it relatively easier to use a variety of experimental diagnostic techniques. Such diagnostics are much more difficult to apply either in the internal thruster flow or in the plume far field. Therefore, understanding the behavior of the thruster plume is critical to the design of thrusters and spacecraft.

A plasma plume is a complex rarefied flow with several species: atoms, positively charged ions, and electrons. Generally, a hybrid particle-fluid approach is used for the computational simulation of plasma-plume flow into vacuum. The direct simulation Monte Carlo (DSMC) method [2] simulates the collisions of heavy particles (ions and atoms), and the particle-in-cell (PIC) technique [3] models the transport of ions in electric fields. Alternatively, a fully kinetic PICVlasov method is developed in which computational particles are 
unequally weighted and periodically redistributed on a local velocity-space grid [4,5]. Electrons are treated using a fluid description, because electrons, which have significantly lighter mass, can adjust their velocities more quickly than ions or atoms.

For the electron fluid model, the Boltzmann relation is usually adopted [6,7]. The Boltzmann model provides the plasma potential using several strong assumptions such as a constant electron temperature for a whole domain. The detailed model, which has been developed recently [8], is based on the conservation laws for electrons and is capable of representing accurate and detailed distributions for electron temperature, plasma potential, and electron velocity. This model was successfully applied in a simulation of an axisymmetric plasma plume from a 200-W-class SPT-type Hall thruster [8] and in another simulation of 3D plasma plumes from a cluster of four 200-W-class Hall thrusters [9].

In this study, the 2D axisymmetric plume flowfields from a D55 TAL Hall thruster are investigated using MONACO [10], a hybrid PIC-DSMC code developed at the University of Michigan with both the Boltzmann model and the detailed model.

The device considered in the present study is the D55 TAL Hall thruster developed by TsNIIMASH. We have chosen to study the D55 Hall thruster because of the availability of a significant amount of experimental data for this device.

Section II reviews numerical models and brief information for experiments and flow conditions. Section III presents general features of the numerical 2D simulation results and a comparison of these results with experimental data taken in the plume of the D55.

\section{Models and Flow Conditions}

\section{A. Plasma Dynamics}

For particle simulation of plume flows, heavy neutrals and ions are modeled with the PIC-DSMC method, and the electrons are assumed as a fluid because electrons adjust their velocities more quickly with their significantly lighter mass.

The simplest fluid-electron model is the Boltzmann relation:

$$
\phi=\phi_{\mathrm{ref}}+\frac{k T_{\mathrm{ref}}}{e} \ln \left(\frac{n_{e}}{n_{\mathrm{ref}}}\right)
$$

This equation is derived using several assumptions, including that the electron flow is isothermal, collisionless, and obeys the ideal gas law, and the magnetic field is neglected.

Recently, the detailed model was proposed [8], which represents a significantly increased level of physics compared with the Boltzmann model. In the detailed model, the electron continuity equation is transformed into a Poisson equation by assuming steady flow and introducing a stream function:

$$
\nabla^{2} \Psi=C_{i} n_{e} n_{a}
$$

where $n_{e} \mathbf{V}_{\mathbf{e}}=\nabla \Psi$, and the xenon ionization rate coefficient $C_{i}$ is expressed as a function of electron temperature using a relation proposed by Ahedo et al. [11]:

$$
C_{i}=\sigma_{i} c_{e}\left(1+\frac{T_{e} \varepsilon_{i}}{\left(T_{e}+\varepsilon_{i}\right)^{2}}\right) \exp \left(-\frac{\varepsilon_{i}}{T_{e}}\right)
$$

The electron momentum equation is given by [12]

$$
\frac{\partial}{\partial t}\left(m_{e} n_{e} \mathbf{V}_{\mathbf{e}}\right)+m_{e} n_{e}\left(\mathbf{V}_{\mathbf{e}} \cdot \nabla\right) \mathbf{V}_{\mathbf{e}}=-e n_{e} \mathbf{E}-\nabla p_{e}+\mathbf{R}
$$

and the friction term is given by

$$
\mathbf{R}=\frac{e n_{e} \mathbf{j}}{\sigma}
$$

Assuming a steady state, neglecting the inertial term on the lefthand side of Eq. (4), a generalized Ohm's law is obtained [8] :

$$
j=\sigma\left[-\nabla \phi+\frac{1}{e n_{e}} \nabla\left(n_{e} k T_{e}\right)\right]
$$

where $-\nabla \phi=\mathbf{E}$.

With given $n_{e}, \mathbf{V}_{\mathrm{e}}, T_{e}$, and the charge continuity condition

$$
\nabla \cdot \mathbf{j}=0
$$

the plasma potential can be obtained []]:

$$
\begin{aligned}
& \nabla \cdot(\sigma \nabla \phi)=\frac{k \sigma}{e}\left(\nabla^{2} T_{e}+T_{e} \nabla^{2}\left(\ln n_{e}\right)\right)+\sigma \nabla\left(\ln n_{e}\right) \cdot \nabla T_{e} \\
& \quad+T_{e} \nabla \sigma \cdot \nabla\left(\ln n_{e}+\nabla \sigma \cdot \nabla T_{e}\right)
\end{aligned}
$$

The electron energy equation is given by [12]

$$
\begin{aligned}
& \frac{\partial}{\partial t}\left(\frac{3}{2} n_{e} k T_{e}\right)+\frac{3}{2} n_{e}\left(\mathbf{V}_{e} \cdot \nabla\right) k T_{e}+p_{e} \nabla \cdot \mathbf{V}_{e} \\
& \quad=\nabla \cdot \kappa_{e} \nabla T_{e}+\mathbf{j} \cdot \mathbf{E}-3 \frac{m_{e}}{m_{i}} v_{e} n_{e} k\left(T_{e}-T_{H}\right)-n_{e} n_{a} C_{i} \varepsilon_{i}
\end{aligned}
$$

Again assuming a steady state, the electron temperature equation is obtained [8]

$$
\begin{gathered}
\nabla^{2} T_{e}=-\nabla \ln \kappa_{e} \cdot \nabla T_{e}+\frac{1}{\kappa_{e}}\left(-\mathbf{j} \cdot \mathbf{E}+\frac{3}{2} n_{e}\left(\mathbf{V}_{e} \cdot \nabla\right) k T_{e}\right. \\
\left.+p_{e} \nabla \cdot \mathbf{V}_{e}+3 \frac{m_{e}}{m_{i}} v_{e} n_{e} k\left(T_{e}-T_{H}\right)+n_{e} n_{a} C_{i} \varepsilon_{i}\right)
\end{gathered}
$$

The electron number density $n_{e}$ is given from the spatial distribution of the ion number density based on the plasma quasineutral assumption.

Finally, the electron conductivity $\sigma$ and the electron thermal conductivity $\kappa_{e}$ can be evaluated using their basic definitions [12]:

$$
\begin{gathered}
\sigma=\frac{e^{2} n_{e}}{m_{e} v_{e}} \\
\kappa_{e}=\frac{2.4}{1+\left(v_{\mathrm{ei}} / \sqrt{2} v_{e}\right)} \frac{k^{2} n_{e} T_{e}}{m_{e} v_{e}}
\end{gathered}
$$

where $v_{e}=v_{\mathrm{ei}}+v_{\mathrm{en}}, v_{\mathrm{ei}}$ is the ion-electron collision frequency, $v_{\mathrm{en}}$ is the neutron-electron collision frequency, and these frequencies are evaluated for the xenon system using cross sections provided in [12].

By treating the right-hand-side terms as known sources and solving Eqs. (2) , ()ㅡ), and (10), three fundamental fluid-electron properties are obtained: that is, electron velocity, plasma potential, and electron temperature. With these detailed properties, the plasmaplume simulation yields much improved results in comparison with the Boltzmann model for the plume of an SPT-type Hall thruster [8]

\section{B. Collision Dynamics}

Two types of collisions are important in the Hall thruster: momentum exchange (MEX) and charge exchange (CEX). There are two kinds of elastic collisions: atom-atom and atom-ion interactions. For atom-atom collisions, the variable hard sphere [2] model is used and the collision cross section for xenon is

$$
\sigma_{\mathrm{el}}(\mathbf{X e}, \mathbf{X e})=\frac{2.12 \times 10^{-18}}{g^{2 \omega}}
$$

where $\omega=0.12$ is related to the viscosity temperature exponent for xenon. For atom-ion elastic collisions, the MEX cross section is set equal to the CEX cross section.

Charge exchange concerns the transfer of one or more electrons between an atom and an ion. For single-charged ions, we use the following cross section measured by Pullins et al. [13] and Miller et al. [14]: 


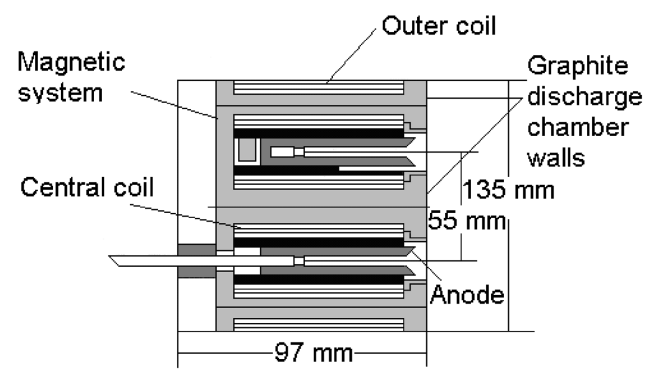

Fig. 1 Schematic of the D55 anode-layer Hall thruster.

$$
\sigma_{\text {cex }}\left(\mathbf{X e}, \mathbf{X e} e^{+}\right)=1.1872 \times 10^{-20}(142.21-23.30 \log (g))
$$

Also, $[13,14]$ reported that the CEX cross section for doublecharged ions is approximately half as large as single-charged ions at corresponding energies.

\section{Flow Conditions}

A schematic of the D55 thruster is presented in Fig. 1. The D55 thruster has an annular anode chamber with a mean diameter of $55 \mathrm{~mm}$ and a width of $5 \mathrm{~mm}$.

We consider three conditions corresponding to three different experiments. Most of the results presented here are for a series of experiments conducted at the University of Michigan $[15,16]$. The D55 thruster was operated at a flow rate of $4.76 \mathrm{mg} / \mathrm{s}$ of xenon, a discharge voltage of $300 \mathrm{~V}$, and a current of $4.5 \mathrm{~A}$. The specific impulse under these conditions was previously measured to be $1810 \mathrm{~s}$ [17]. When a thruster exit velocity is chosen consistent with the plasma potential and laser-induced fluorescence (LIF) data, this corresponds to the case in which about $80 \%$ of the thrust is generated inside the thruster. The number fraction of double-xenon ions is assumed to be 0.25 . At the thruster exit, the electron temperature is taken to be $10 \mathrm{eV}$ in the Boltzmann model and the detailed model to obtain good agreement between the simulations and the data measured in the plume field, the temperature of the ions is assumed to be $4 \mathrm{eV}$, and that of the neutrals is assumed to be $750 \mathrm{~K}$. The backpressure in the Michigan facility is reported as $8.3 \times 10^{-3} \mathrm{~Pa}$.

The second flow condition investigated corresponds to a study performed by TsNIIMASH [18]. The thruster was operated at a flow rate of $3.5 \mathrm{mg} / \mathrm{s}$ and a current of $3 \mathrm{~A}$. The background pressure with the thruster running was $5.9 \times 10^{-3} \mathrm{~Pa}$.

The third flow condition corresponds to a study performed by the University of Tennessee Space Institute (UTSI) and Lockheed Martin Astronautics (LMA) [19]. The thruster was operated at a flow rate of $6 \mathrm{mg} / \mathrm{s}$ and a current of $4.5 \mathrm{~A}$. The background pressure with the thruster running was $9.3 \times 10^{-3} \mathrm{~Pa}$.

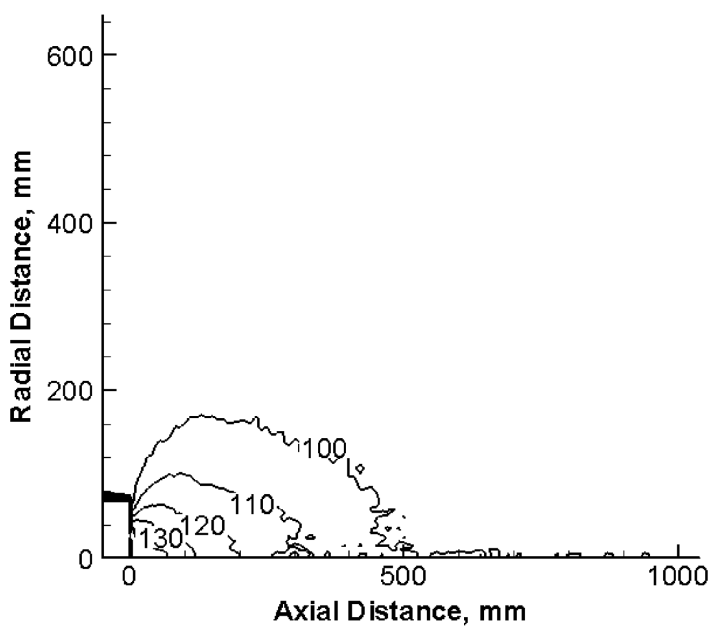

a) The Boltzmann model
Table 1 Flow properties assumed at the thruster exit plane

\begin{tabular}{lccrr}
\hline \hline Experiment & Species & $n, \mathrm{~cm}^{-3}$ & \multicolumn{1}{c}{$T, \mathrm{~K}$} & $U, \mathrm{~m} / \mathrm{s}$ \\
\hline Michigan & $\mathrm{Xe}$ & $3.8 \times 10^{12}$ & 750 & 281 \\
& $\mathrm{Xe}^{+}$ & $3.6 \times 10^{11}$ & 46,400 & 15,000 \\
& $\mathrm{Xe}^{++}$ & $9.0 \times 10^{10}$ & 46,400 & 21,300 \\
TsNIIMASH & $\mathrm{Xe}$ & $4.6 \times 10^{12}$ & 750 & 281 \\
& $\mathrm{Xe}^{+}$ & $2.4 \times 10^{11}$ & 46,400 & 15,000 \\
& $\mathrm{Xe}^{++}$ & $6.0 \times 10^{10}$ & 46,400 & 21,300 \\
UTSI and LMA & $\mathrm{Xe}^{13}$ & $1.2 \times 10^{13}$ & 750 & 281 \\
& $\mathrm{Xe}^{+}$ & $3.6 \times 10^{11}$ & 46,400 & 15,000 \\
& $\mathrm{Xe}^{++}$ & $9.0 \times 10^{10}$ & 46,400 & 21,300 \\
\hline \hline
\end{tabular}

The D55 has a nozzlelike geometry at the exit, and so the plume spreads at the thruster exit with certain angles. In the present study, we adopt $15 \mathrm{deg}$ as a half-angle, and the radial velocity varies linearly across each half of the exit plane [7].

The computational grid employed in the present study consists of rectangular cells. The smallest cells are located close to the thruster exit and have a size of $5 \mathrm{~mm}$. The largest cells are those close to the edges of the domain and have a size of $1 \mathrm{~cm}$. The computations presented in the study typically employed 500,000 particles with a total of 60,000 time steps. Table 1 is a listing of the flow conditions assumed at the thruster exit.

\section{Results}

Overall plasma potential fields obtained with the Boltzmann model and the detailed model are presented in Figs. 2a and 2b, respectively. For both models, we set $145 \mathrm{~V}$ as the plasma potential at the channel exit to get good agreement between measured and simulated data, especially radial profiles of ion current density and axial components of velocity in the very near-field plume.

It is known that the plasma potential fields of the Boltzmann model and the detailed model are very different [ㅁ]. We can see similar features in Figs. $2 \mathrm{a}$ and $2 \mathrm{~b}$. The Boltzmann model gives weaker gradients in plasma potential, and the overall variation in potential is only about $30 \mathrm{~V}$.

By comparison, the potential gradients and electric fields associated with the detailed model are much stronger, with a total variation in potential of about $140 \mathrm{~V}$. Hence, the detailed model produces significantly greater ion acceleration. This feature will be discussed later.

A series of probe experiments was performed by Domonkos et al. [15] in the near field of the D55 plume. The local plasma potential was obtained using an emissive probe and a Langmuir probe, ion current density was obtained using a Faraday probe, and the electron temperature and number density were obtained using a Langmuir probe. Figures $\underline{3 \mathrm{a}}$ and $\underline{3 \mathrm{~b}}$ show radial profiles of plasma potential at

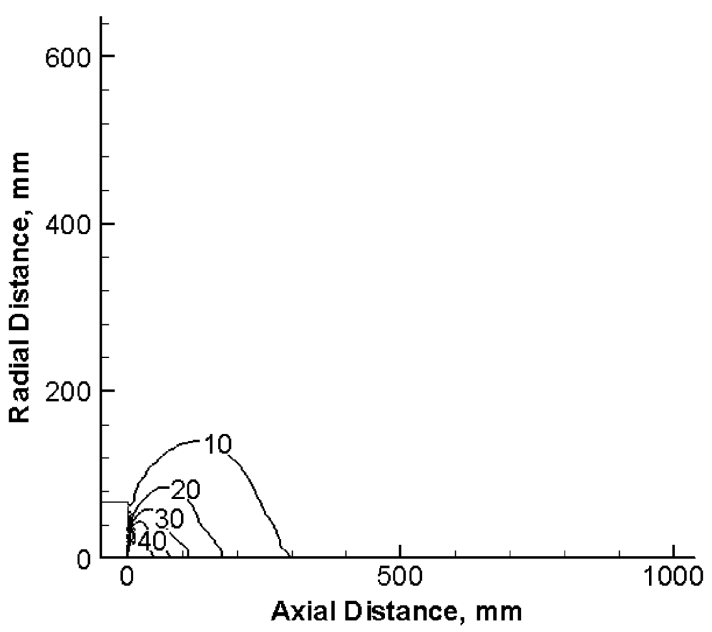

b) The detailed model

Fig. 2 Plasma potential (V) profiles. 


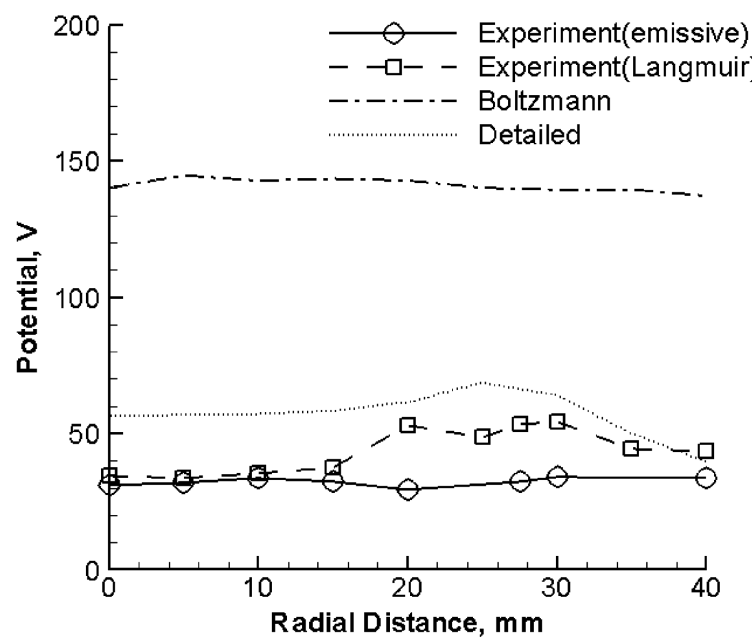

a) $10 \mathrm{~mm}$ from the thruster exit plane

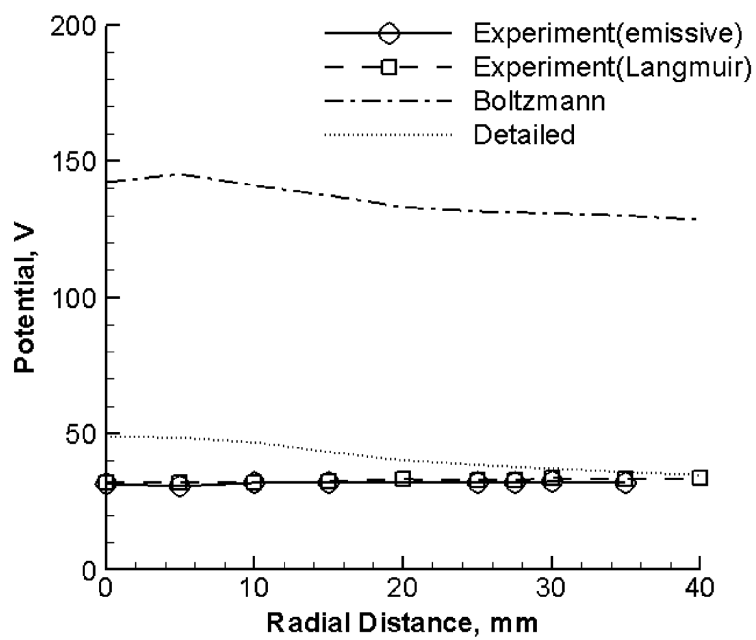

b) $50 \mathrm{~mm}$ from the thruster exit plane

Fig. 3 Radial profiles of plasma potential.

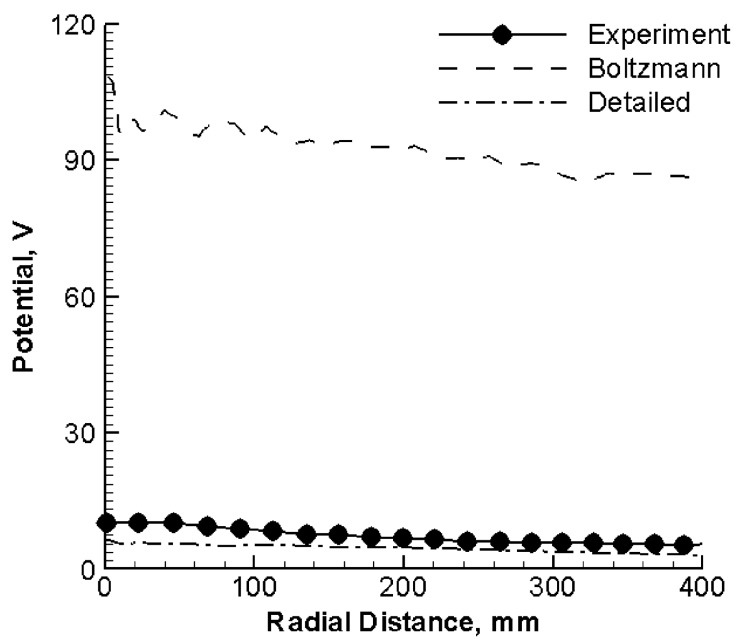

Fig. 4 Radial profiles of plasma potential at $500 \mathrm{~mm}$ from the thruster exit plane.

axial distances of 10 and $50 \mathrm{~mm}$ from the thruster exit plane, respectively. Data were measured with respect to the cathode potential of $14 \mathrm{~V}$, and so here we added $14 \mathrm{~V}$ to the measured data for consistency with the simulation. Close to the thruster, the Boltzmann model and the detailed model overpredict the potential. At $10 \mathrm{~mm}$, the detailed model shows the plasma potential increasing in the region of the discharge chamber, which is at $20 \mathrm{~mm} \leq r \leq 35 \mathrm{~mm}$, and we can see that the detailed model captures the shape quite well. At $50 \mathrm{~mm}$ from the thruster, the Boltzmann model still greatly overpredicts the potential, but the detailed model results and measured data are in better agreement.

Comparisons between measured data and simulation results for the potential in the far-field plume are shown in Fig. 4. The measured data were obtained by Zakharenkov et al. [18] and the simulations use the second set of operating conditions given in Table 1 . At a distance of $500 \mathrm{~mm}$ from the thruster, the Boltzmann model again greatly overpredicts the potential, whereas the detailed model reproduces fairly well the measured profiles.

Ion-current-density profiles predicted by the simulation are compared with the experimental data [15] in Figs. 5a and $\underline{5 b}$ along radial lines located 10 and $40 \mathrm{~mm}$ from the thruster exit plane, respectively. The Boltzmann model greatly underpredicts the measured values at $40 \mathrm{~mm}$, but the detailed model shows excellent agreement with experimental measurement. The variation of the ion current density with axial distance from the thruster indicates that the ion flow begins as an annulus and then merges into a cylinder-shaped beam. This feature emerges because the annular ion flow is diverging with an angle of $15 \mathrm{deg}$ so that an overlap occurs at the centerline of the thruster as the flow convects downstream.

Figure 6 shows further comparisons between measured data [18] and simulation results for ion current density in the far-field plume.

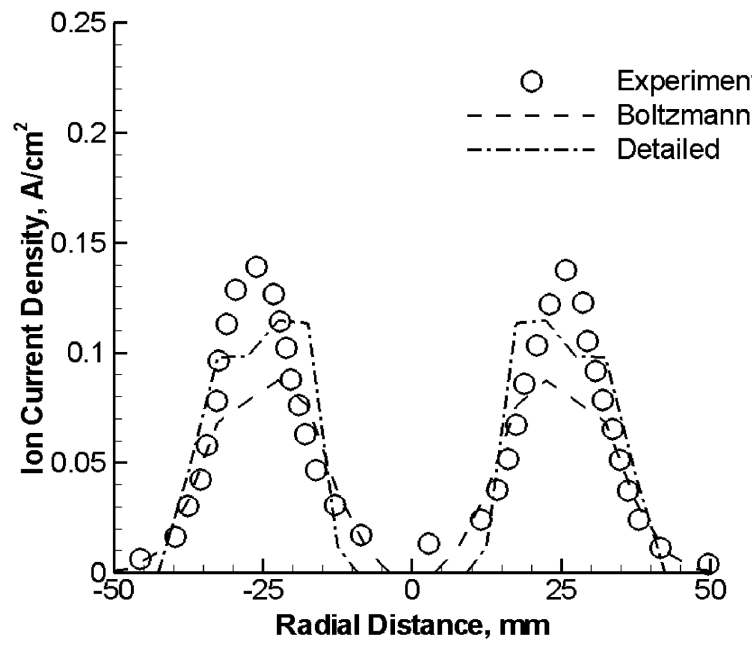

a) $10 \mathrm{~mm}$ from the thruster exit plane

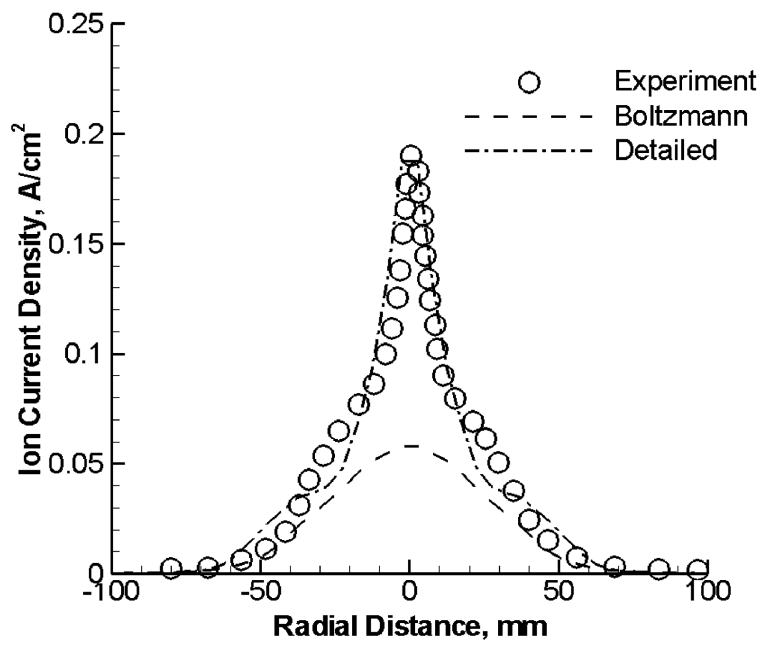

b) $40 \mathrm{~mm}$ from the thruster exit plane

Fig. 5 Radial profiles of ion current density. 


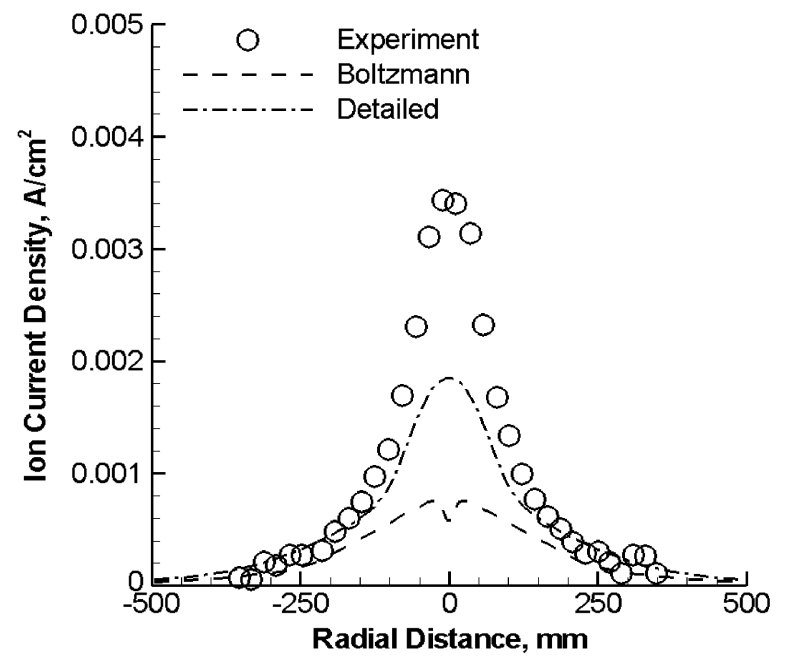

Fig. 6 Radial profiles of ion current density at $500 \mathrm{~mm}$ from the thruster exit plane.

The detailed model shows better agreement with measurements, though both models underpredict the measured values. This underprediction of the current density over the entire range implies a possibility that the simulation overaccelerates ionized particles in the radial direction. This feature is consistent with the comparison of electron number density. This feature is discussed later.

Measurements of electron number density [15] are compared with the simulations for radial profiles at 10 and $50 \mathrm{~mm}$ in Figs. $7 \mathrm{a}$ and $7 \mathrm{~b}$, respectively. The simulation values represent the total charge density obtained from the number densities of the $\mathrm{Xe}^{+}$and $\mathrm{Xe}^{++}$ions. The measured data have an accuracy of $\pm 50 \%$ at 10 and $50 \mathrm{~mm}$. Most of the simulation data are within the range at $z=10 \mathrm{~mm}$, although they show a tendency to underpredict the measured values. At $z=40 \mathrm{~mm}$, both simulation data sets coincide with the measurement around the centerline but underestimate the electron number density as $r$ increases. The peak electron number density measured at both stations is more than double the total charge density assumed in the simulations at the thruster exit plane (see Table 1). Gulczinski et al. [16] presented evidence that the Langmuir probe technique leads to high electron number densities. At distances of 25 and $50 \mathrm{~cm}$ from the thruster, the Langmuir probe gave six-times-higher electron number density than the microwave interferometer [16].

Further comparisons between measured data and simulation results for electron number density in the far-field plume are shown in Figs. $8 \mathrm{a}$ and $8 \mathrm{~b}$. The measured data were obtained by Gulczinski et al. [16] using microwave interferometry, and the simulations use the first set of operating conditions given in Table 1 . The uncertainty for these data is $\pm 10 \%$. The detailed model shows better agreement with measurements, though both models still underpredict the measured values over the entire radial profile. One possible reason for these

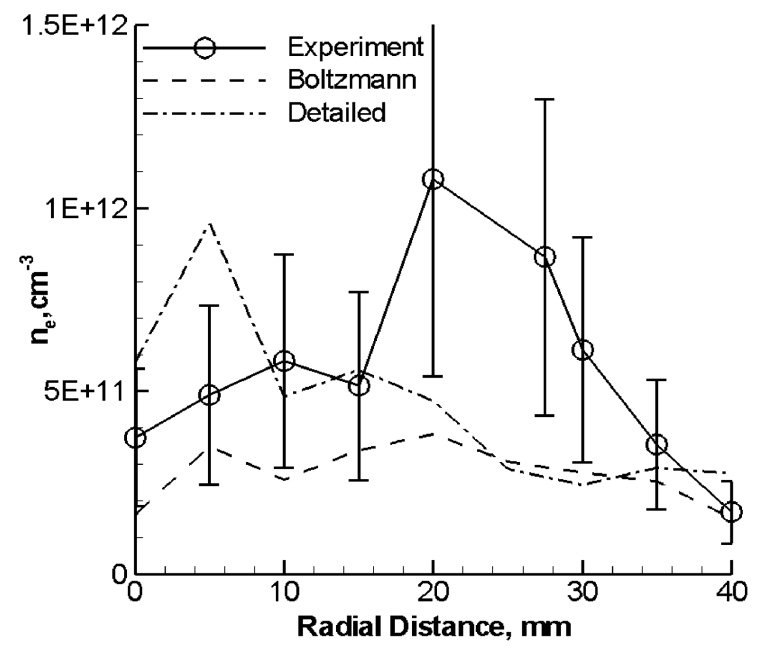

a) $10 \mathrm{~mm}$ from the thruster exit plane

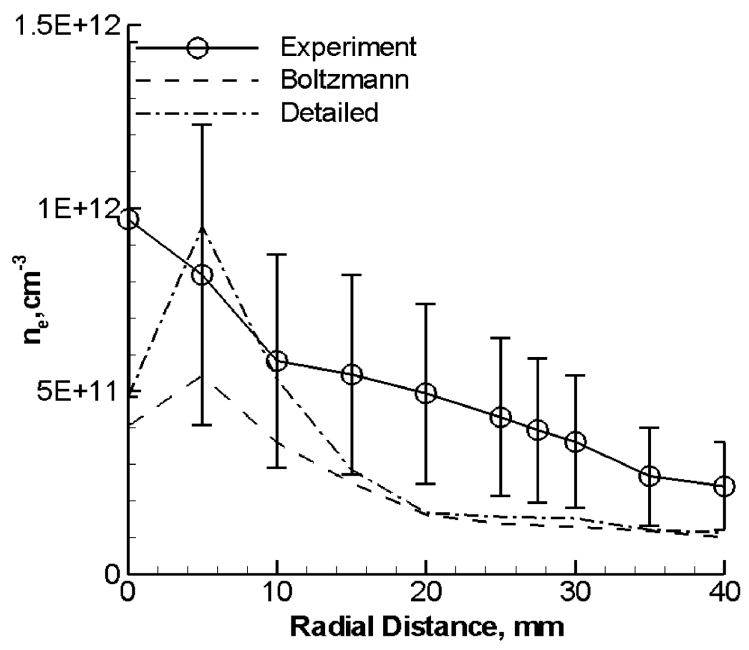

b) $50 \mathrm{~mm}$ from the thruster exit plane

Fig. 7 Radial profiles of electron number density.

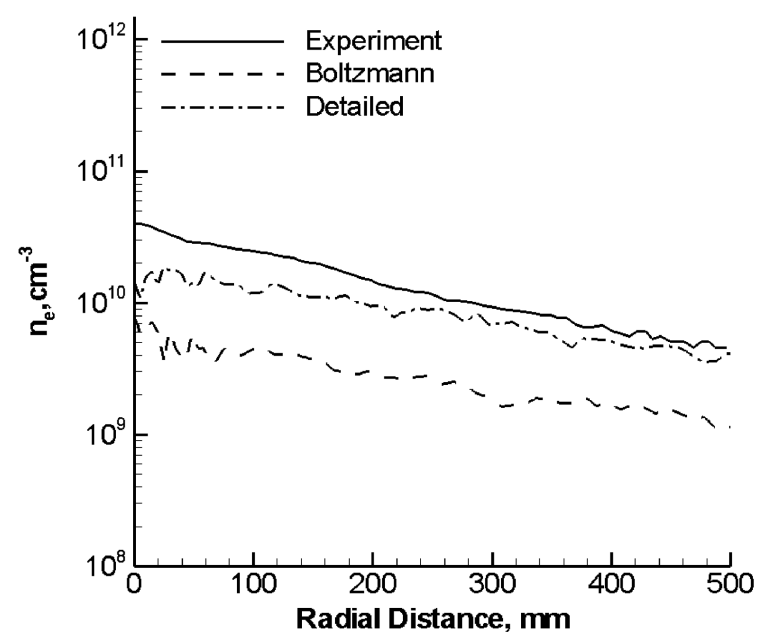

a) $500 \mathrm{~mm}$ from the thruster exit plane

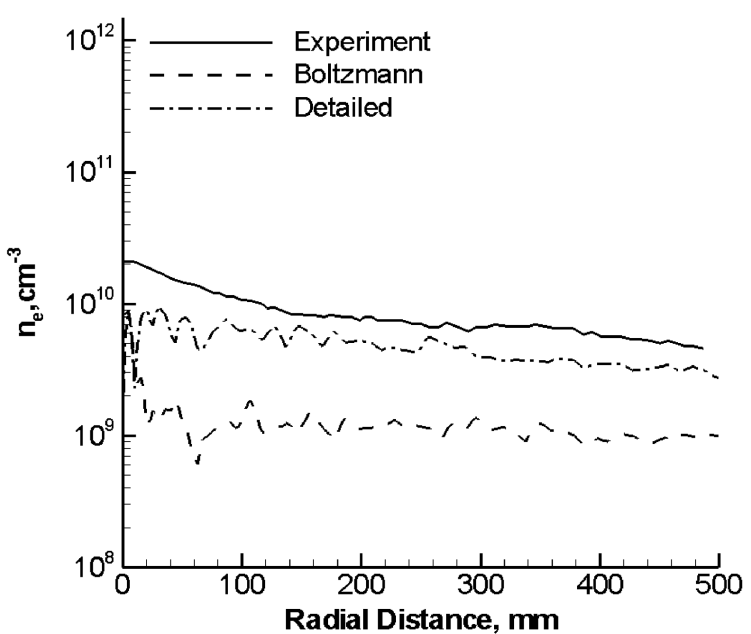

b) $1000 \mathrm{~mm}$ from the thruster exit plane

Fig. 8 Radial profiles of electron number density. 


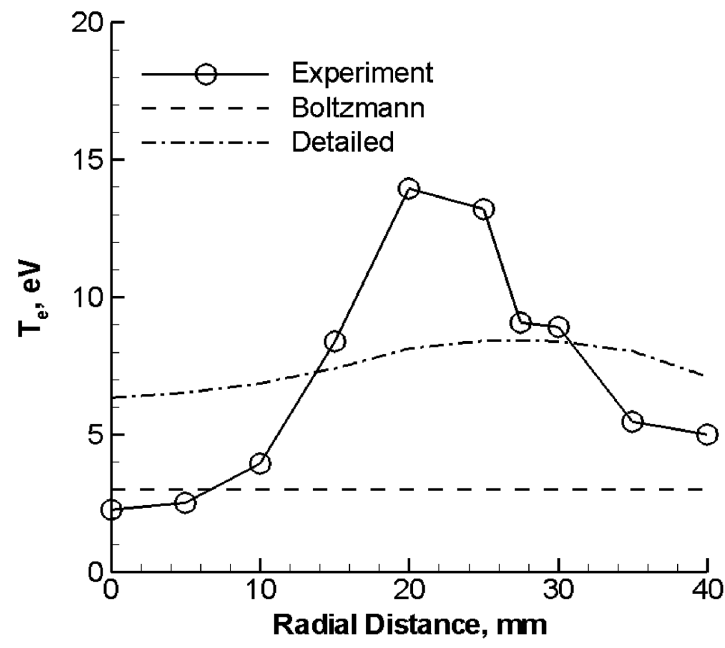

a) $10 \mathrm{~mm}$ from the thruster exit plane

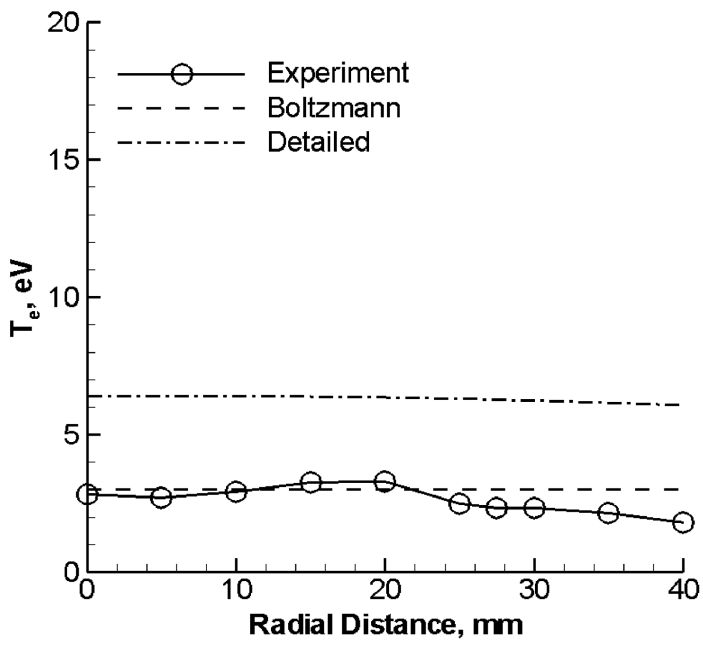

b) $50 \mathrm{~mm}$ from the thruster exit plane

Fig. 9 Radial profiles of electron temperature.

differences between the detailed model results and the experiment is that the electric fields in the simulation accelerate the ions too much in both the axial and radial directions. It is known that the magnetic field leaked from the thruster is strong enough to affect the electron motion in the near-field plume region [20]. So one way to address this difference would be to include partial confinement of electrons caused by the magnetic field of the thruster leaking into the near-field plume. This idea is consistent with the comparison of far-field potential shown in Fig. 4, in which the simulation predicts lower values than those measured.

Figures $9 \mathrm{a}$ and $9 \mathrm{~b}$ show radial profiles of electron temperature at distances of 10 and $50 \mathrm{~mm}$ from the thruster, respectively. The experimental uncertainty is reported to be $\pm 10 \%$ [15]. In general, although the detailed model provides reasonable agreement with the measurements, the radial gradients predicted by the model are smaller than the measured data indicate.

Far-field predictions of the electron temperature profiles are presented in Fig. 10. The measured data were obtained by Zakharenkov et al. [18], and the simulations use the second set of operating conditions given in Table 1 . It is clearly shown that the detailed model gives good agreement with the measured data in the far field.

The simulation results are compared with LIF measurements of $\mathrm{Xe}^{+}$axial velocity component obtained by Keefer et al. [19] in the near-field plume. In [19], it is explained that the reported velocity data represent the central value of the ion velocity distribution functions detected by the LIF diagnostic. Therefore, for consistency

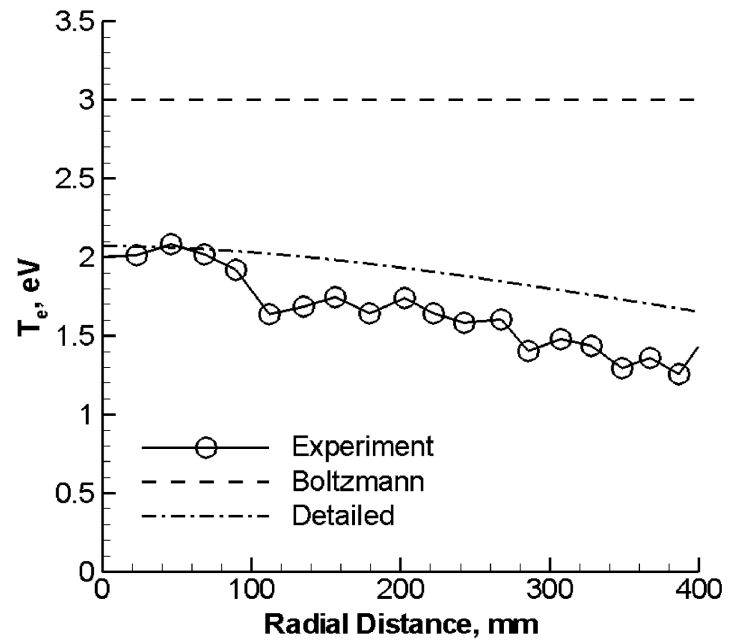

Fig. 10 Radial profiles of electron temperature at an axial distance of $500 \mathrm{~mm}$ from the thruster. with the experiment, the ion velocity distribution function is calculated throughout the flowfield, and the most probable value of the distributions is obtained. Figure 11 shows the axial velocity profiles at a radial position of $27.5 \mathrm{~mm}$, which is along the thruster channel center. The simulations use the third set of operating conditions given in Table 1. It is clear that the Boltzmann model fails to produce sufficient ion acceleration in the near field of the plume. As discussed with reference to Figs. $2 a$ and $2 b$, the detailed model predicts strong ion acceleration in the near-field region and rapidly accelerates the ions from the thruster exit velocity of $15 \mathrm{~km} / \mathrm{s}$ to a value of about $20 \mathrm{~km} / \mathrm{s}$ that corresponds to the measured data. The simulation result, however, overestimates the axial velocity at $z=1 \mathrm{~mm}$

Finally, results obtained with the Boltzmann model and the detailed model are compared for different thruster exit potentials. Figures 12 and 13 show that the Boltzmann model cannot provide reasonable profiles of plasma potential and ion velocity simultaneously. Figure 12 presents radial profiles of plasma potential at $500 \mathrm{~mm}$ from the thruster, and Fig. 13 provides the axial components of ion velocity. In Fig. 12, one can see that a potential can be chosen that is consistent with far-field measurements. Figure 13, however, shows that this low potential gives an ion velocity that is much higher than the measurements. It is possible to choose a reasonable ion velocity in Fig. 13, but in that case, it can be shown from Fig. 12 that the plasma potentials are much higher than the measurements. The Boltzmann model can only give agreement with the measured potential by assuming an ion velocity of $20 \mathrm{~km} / \mathrm{s}$

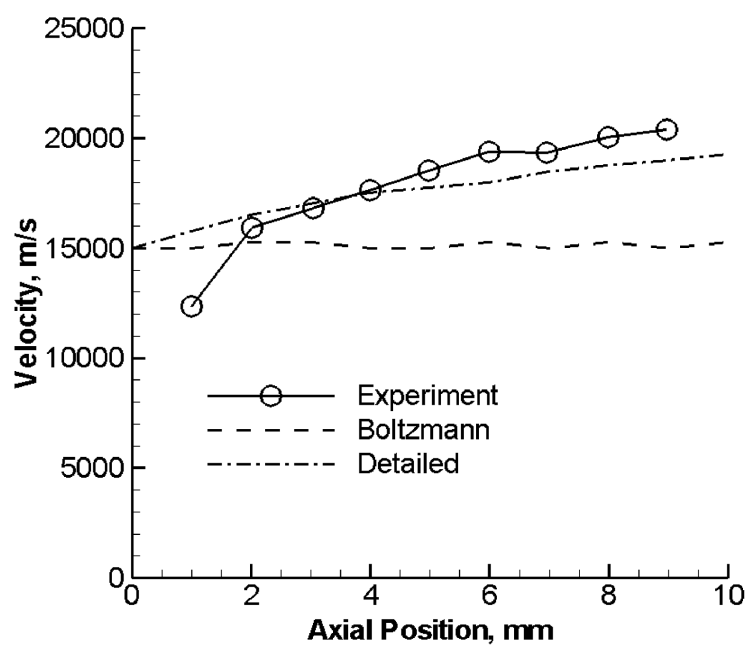

Fig. 11 Axial components of velocity at a radial position of $27.5 \mathrm{~mm}$. 


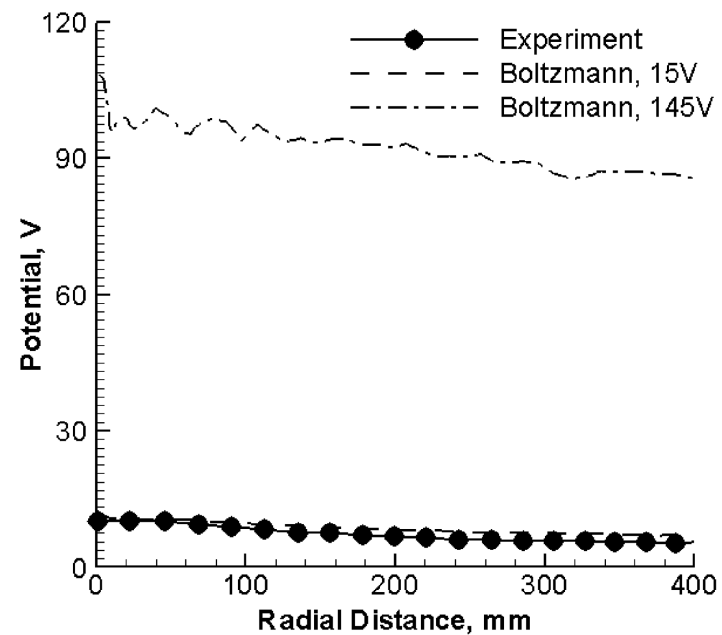

Fig. 12 Radial profiles of plasma potential at $500 \mathrm{~mm}$ from the thruster exit plane.

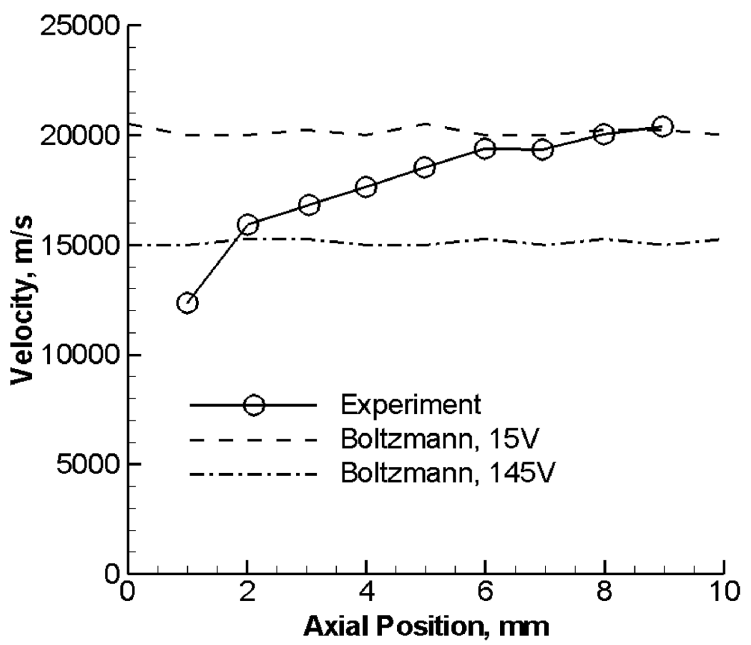

Fig. 13 Axial components of velocity at a radial position of $27.5 \mathrm{~mm}$.

at the thruster exit, which is inconsistent with the LIF measurement [19]. Thus, for this thruster, the Boltzmann model only gives good agreement with the plasma potential by assuming an erroneously high thruster exit ion velocity. Figures 14 and 15 show that there is also a need to improve the detailed model. Figure 14 shows radial profiles of plasma potential at $10 \mathrm{~mm}$ from the thruster, and Fig. 15

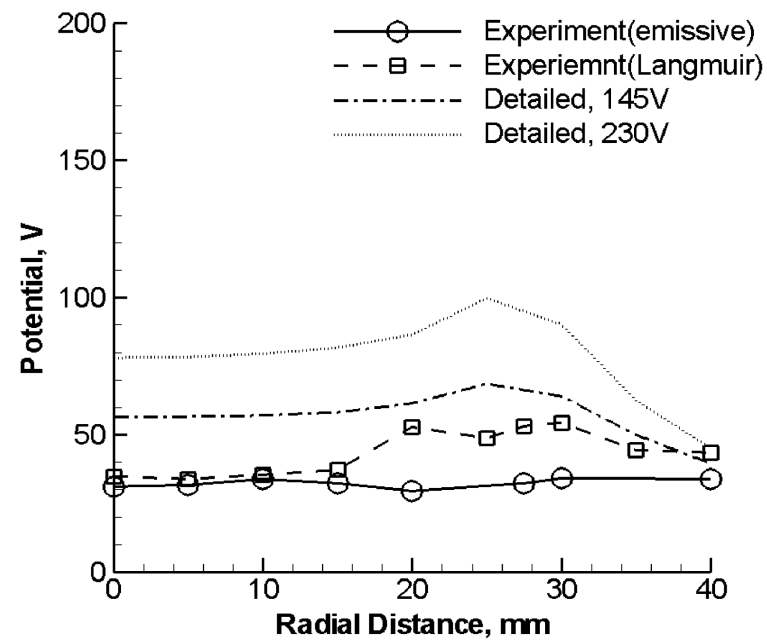

Fig. 14 Radial profiles of plasma potential at $10 \mathrm{~mm}$ from the thruster exit plane.

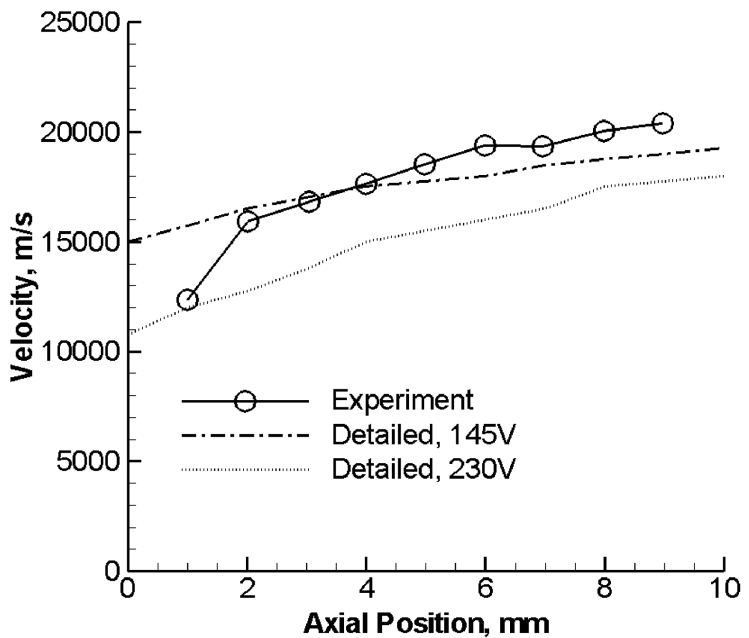

Fig. 15 Axial components of velocity at a radial position of $27.5 \mathrm{~mm}$.

provides the axial components of ion velocity. In Fig. 14, we can see that a plasma potential can be chosen that is consistent with the measurement, but Fig. 15 shows that the ion velocity is too high right beside the thruster with this plasma potential. Also, Fig. 15 shows that when the ion velocity is chosen to be consistent with the LIF measurement, then the potentials are too high in Fig. 14. Possible explanations for the problems with the detailed model include the simple thruster exit conditions employed and the omission of the magnetic field effects on both the electrons and ions.

\section{Conclusions}

A hybrid particle-fluid PIC-DSMC model using both the detailed model and the Boltzmann model for the fluid electrons was applied to simulate the plume flow from a D55 anode-layer Hall thruster. Generally, the detailed model provided better results than the Boltzmann model. The detailed model accurately predicted the extended ion acceleration region outside the thruster. By comparison, the Boltzmann model indicated almost no ion acceleration outside the thruster. The simulation results of the detailed model and the Boltzmann model underpredicted the electron number density, especially in the far-plume field. This disparity may be caused by the simulated electric fields being too strong and leading to overacceleration of the ions in the radial direction. One possible mechanism that should be included in future work is partial confinement of electrons by the magnetic field of the thruster leaking into the plume. Future work will also involve modeling the D55 thruster plasma to generate improved thruster exit flow conditions.

\section{Acknowledgments}

This work is supported by NASA grant NNC04CB15C and grant FA9550-05-1-0042 from the U.S. Air Force Office of Scientific Research. The authors gratefully acknowledge the contributions to this work by discussions with Chunpei Cai.

\section{References}

[1] Marrese-Reading, C. M., Frisbee, R., Sengupta, A., Cappelli, M. A., Tverdoklebov, S., Semenkin, S., and Boyd, I. D., "Very High $I_{\mathrm{sp}}$ Thruster with Anode Layer (VHITAL): An Overview," AIAA Paper 2004-5910, Sept. 2004.

[2] Bird, G. A., Molecular Gas Dynamics and the Direct Simulation of Gas Flows, Oxford Press, New York, 1994.

[3] Birdsall, C. K., and Langdon, A. B., Plasma Physics Via Computer Simulation, Adam Hilger, New York, 1991.

[4] Batishchev, O., "Hybrid PIC-Vlasov Method," 28th IEEE International Conference on Plasma Science, Vol. 1, Inst. of Electrical and Electronics Engineers, Piscataway, NJ, 2001, pp. 565-568.

[5] Fox, J. M., Batishcheva, A. A., Batishchev, O. V., and MartinezSanchez, M., "Adaptively Meshed Fully Kinetic PIC-Vlasov 
Model for Near Vacuum Hall Thrusters,” AIAA Paper 2006-4324, July 2006.

[6] Keidar, M., Choi, Y., and Boyd, I. D., "Modeling a Two-Stage HighPower Bismuth Anode Layer Thruster and its Plume," International Electric Propulsion Conference Paper 2005-045, Oct. 2005.

[7] Boyd, I. D., "Computation of the Plume of an Anode-Layer Hall Thruster," Journal of Propulsion and Power, Vol. 16, No. 5, 2000, pp. 902-909.

[8] Boyd, I. D., and Yim, J. T., "Modeling of the Near Field Plume of a Hall Thruster," Journal of Applied Physics, Vol. 95, May 2004, pp. 45754584.

doi:10.1063/1.1688444

[9] Cai, C., and Boyd, I. D. " "3D Simulation of Plume Flows from a Cluster of Plasma Thrusters," AIAA Paper 2005-4662, July 2005.

[10] Dietrich, S., and Boyd, I. D., "Scalar and Parallel Optimized Implementation of the Direct Simulation Monte Carlo Method," Journal of Computational Physics, Vol. 126, July 1996, pp. 328-342. doi:10.1006/jcph.1996.0141

[11] Ahedo, E., Martinez-Cerezo, P., and Martinez-Sanchez, M., "OneDimensional Model of the Plasma Flow in a Hall Thruster," Physics of Plasmas, Vol. 8, June 2001, pp. 3058-3068. doi:10.1063/1.1371519

[12] Mitcher, M., and Kruger, C. H., Partially Ionized Gases, Wiley, New York, 1973.

[13] Pullins, S. H., Chiu, Y., Levandier, D. J., and Dresseler, R. A., "Ion Dynamics in Hall Effect and Ion Thruster-Xenon Symmetric Charge Transfer," AIAA Paper 2000-0636, Jan. 2000.
[14] Miller, S., Levandier, D. J., Chiu, Y., and Dresseler, R. A., "Xenon Charge Exchange Cross Sections for Electrostatic Thruster Models," Journal of Applied Physics, Vol. 91, Feb. 2002, pp. 984-991. doi:10.1063/1.1426246

[15] Domonkos, M. T., Marrese, C. M., Haas, J. M., and Gallimore, A. D., "Very Near-Field Plume Investigations of the D55," AIAA Paper 973062, July 1997.

[16] Gulczinski, F. S., Gallimore, A. D., Carlson, D. O., and Gilchrist, B. E., "Impact of Anode Layer Thruster Plumes on Satellite Communications," AIAA Paper 97-3067, July 1997.

[17] Semenkin, A., Kochergin, A., Garkusha, V., Chislov, G., Rusakov, A., Tverdoklebov, S., and Sota, C., "RHETT/EPDM Flight Anode Layer Thruster Development," IEPC Paper 97-106, August 1997.

[18] Zakharenkov, L., Semenkin, A. V., and Lebedev, Y. V., "Measurement Features and Results of TAL D-55 Plume," International Electric Propulsion Conference Paper 2005-184, Oct. 2005.

[19] Keefer, D., Wright, N., Hornkohl, J. O., and Bangasser, J., "Multiplexed LIF and Langmuir Probe Diagnostic Measurements in the TAL D-55 Thruster," AIAA Paper 97-2425, July 1999.

[20] Keidar, M., and Boyd, I. D., "Effect of a Magnetic Field on the Plasma Plume from Hall Thrusters," Journal of Applied Physics, Vol. 86, No. 9 , 1999, pp. 4786-4791. doi: $10.1063 / 1.371444$

E. Choueiri Associate Editor 